\title{
JOSEPH IDEALS AND LISSE MINIMAL $W$-ALGEBRAS
}

\author{
TOMOYUKI ARAKAWA AND ANNE MOREAU
}

\begin{abstract}
We consider a lifting of Joseph ideals for the minimal nilpotent orbit closure to the setting of affine Kac-Moody algebras and find new examples of affine vertex algebras whose associated varieties are minimal nilpotent orbit closures. As an application we obtain a new family of lisse $\left(C_{2}\right.$-cofinite $) W$ algebras that are not coming from admissible representations of affine KacMoody algebras.
\end{abstract}

\section{INTRODUCTION}

Let $\mathfrak{g}$ be a finite-dimensional simple Lie algebra over $\mathbb{C},(\mid)$ be a normalized invariant inner product, i.e., $\frac{1}{2 h^{\vee}} \times$ Killing form.

Let $\widehat{\mathfrak{g}}=\mathfrak{g}\left[t, t^{-1}\right] \oplus \mathbb{C} K \oplus \mathbb{C} D$ be the Kac-Moody Lie algebra associated with $\mathfrak{g}$ and $(\mid)$, with the commutation relations

$$
\begin{array}{r}
{[x(m), y(n)]=[x, y](m+n)+m(x \mid y) \delta_{m+n, 0} K,} \\
{[D, x(m)]=m x(m), \quad[K, \widehat{\mathfrak{g}}]=0,}
\end{array}
$$

where $x(m)=x \otimes t^{m}$. For $k \in \mathbb{C}$, set

$$
V^{k}(\mathfrak{g})=U(\widehat{\mathfrak{g}}) \otimes_{U(\mathfrak{g}[t] \oplus \mathbb{C} K \oplus \mathbb{C} D)} \mathbb{C}_{k},
$$

where $\mathbb{C}_{k}$ is the one-dimensional representation of $\mathfrak{g}[t] \oplus \mathbb{C} K \oplus \mathbb{C} D$ on which $\mathfrak{g}[t] \oplus D$ acts trivially and $K$ acts as multiplication by $k$. The space $V^{k}(\mathfrak{g})$ is naturally a vertex algebra, and it is called the universal affine vertex algebra associated with $\mathfrak{g}$ at level $k$. By the PBW theorem, $V^{k}(\mathfrak{g}) \cong U\left(\mathfrak{g}\left[t^{-1}\right] t^{-1}\right)$ as $\mathbb{C}$-vector spaces.

Let $V_{k}(\mathfrak{g})$ be the unique simple graded quotient of $V^{k}(\mathfrak{g})$. As a $\widehat{\mathfrak{g}}$-module, $V_{k}(\mathfrak{g})$ is isomorphic to the irreducible highest weight representation of $\widehat{\mathfrak{g}}$ with highest weight $k \Lambda_{0}$, where $\Lambda_{0}$ is the dual element of $K$.

Let $X_{V}$ be the associated variety [Ar12] of a vertex algebra $V$, which is the maximum spectrum of Zhu's $C_{2}$-algebra,

$$
R_{V}:=V / C_{2}(V) .
$$

In the case $V$ is a quotient of $V^{k}(\mathfrak{g}), V / C_{2}(V)=V / \mathfrak{g}\left[t^{-1}\right] t^{-2} V$ and we have a surjective Poisson algebra homomorphism

$$
\mathbb{C}\left[\mathfrak{g}^{*}\right]=S(\mathfrak{g}) \rightarrow V / \mathfrak{g}\left[t^{-1}\right] t^{-2} V, \quad x \mapsto \overline{x(-1)}+\mathfrak{g}\left[t^{-1}\right] t^{-2} V,
$$

2010 Mathematics Subject Classification. 17B67, 17B69, 81R10.

Key words and phrases. Joseph ideal, associated variety, Deligne exceptional series, affine Kac-Moody algebra, affine $W$-algebra. 
where $\overline{x(-1)}$ denotes the image of $x(-1)$ in the quotient $V$. Then $X_{V}$ is just the zero locus of the kernel of the above map in $\mathfrak{g}^{*}$. It is $G$-invariant and conic, where $G$ is the adjoint group of $\mathfrak{g}$. Note that on the contrary to the associated variety of a primitive ideal of $U(\mathfrak{g})$, the variety $X_{V_{k}(\mathfrak{g})}$ is not necessarily contained in the nilpotent cone $\mathcal{N}$ of $\mathfrak{g}$. In fact, $X_{V_{k}(\mathfrak{g})}=\mathfrak{g}^{*}$ for a generic $k$ since $V_{k}(\mathfrak{g})=V^{k}(\mathfrak{g})$ in this case.

A conjecture of Feigin and Frenkel, proved in [Ar15a], states that $X_{V_{k}(\mathfrak{g})} \subset \mathcal{N}$ if $V_{k}(\mathfrak{g})$ is admissible [KW89]. In fact it is also believed that the converse is true, that is, $X_{V_{k}(\mathfrak{g})} \subset \mathcal{N}$ only if $V_{k}(\mathfrak{g})$ is admissible, so that the condition $X_{V_{k}(\mathfrak{g})} \subset \mathcal{N}$ gives a geometric characterization of admissible affine vertex algebras. One of the aims of this paper is to provide a counterexample of this fact, that is, there exist non-admissible affine vertex algebras $V_{k}(\mathfrak{g})$ such that $X_{V_{k}(\mathfrak{g})} \subset \mathcal{N}$.

Let $\left(e_{\theta}, h_{\theta}, f_{\theta}\right)$ be the $\mathfrak{s l}_{2}$-triple associated with the highest positive root $\theta$ of $\mathfrak{g}$. Let $\mathbb{O}_{\text {min }}=G$. $f_{\theta}$ be the unique minimal non-trivial nilpotent orbit of $\mathfrak{g}$ which is of dimension $2 h^{\vee}-2$, [W99], where $h^{\vee}$ is the dual Coxeter number of $\mathfrak{g}$.

Consider the Deligne exceptional series

$$
A_{1} \subset A_{2} \subset G_{2} \subset D_{4} \subset F_{4} \subset E_{6} \subset E_{7} \subset E_{8}
$$

discussed in [D96, DG02].

Theorem 1.1. (1) Assume that $\mathfrak{g}$ belongs to the Deligne exceptional series and that

$$
k=-\frac{h^{\vee}}{6}-1
$$

Then $X_{V_{k}(\mathfrak{g})}=\overline{\mathbb{O}_{\text {min }}}$.

(2) Assume that $\mathfrak{g}$ is of type $D_{4}, E_{6}, E_{7}, E_{8}$ and that $k$ is an integer such that

$$
-\frac{h^{\vee}}{6}-1 \leqslant k \leqslant-1 \text {. }
$$

Then $X_{V_{k}(\mathfrak{g})}=\overline{\mathbb{O}_{\text {min }}}$.

(3) Assume that $\mathfrak{g}$ is of type $D_{l}, l \geqslant 5$. Then $X_{V_{k}(\mathfrak{g})}=\overline{\mathbb{O}_{\text {min }}}$ for $k=-2,-1$.

Note that for $\mathfrak{g}$ of type $A_{1}, A_{2}, G_{2}, F_{4}$, the rational number $-h^{\vee} / 6-1$ is admissible. However for types $D_{4}, E_{6}, E_{7}, E_{8}$, the number $-h^{\vee} / 6-1$ is a negative integer which is certainly non-admissible ([KW08, Proposition 1.2]).

A consequence of the fact $X_{V_{k}(\mathfrak{g})} \subset \mathcal{N}$ is that $V_{k}(\mathfrak{g})$ has only finitely many simple modules in the category $\mathcal{O}$ (cf. Corollary 5.3), as in case $V_{k}(\mathfrak{g})$ is admissible [Ad94, AM95, Ad97, Pe07a, Pe07b, AL11, Ar16]. If $\mathfrak{g}$ belongs to the Deligne exceptional series outside the type $A$ and $k=-h^{\vee} / 6-1$, it is possible to derive the classification of simple $V_{k}(\mathfrak{g})$-modules that belong to $\mathcal{O}$ from Joseph's result [J98] in the following manner.

If $\mathfrak{g}$ is not of type $A$, it is known [J76, GS04] that there exists a unique completely prime ideal $\mathcal{J}_{0}$ in $U(\mathfrak{g})$, called the Joseph ideal, whose associated variety is $\overline{\mathbb{O}_{\text {min }}}$, that is, $\overline{\mathbb{O}_{\text {min }}}$ is the zero locus in $\mathfrak{g}^{*}$ of gr $\mathcal{J}_{0}$. As a by-product, we obtain a lifting to the Joseph ideal in the following sense. For a $\mathbb{Z}_{\geqslant 0}$-graded vertex algebra $V$, let $A(V)$ be its Zhu's algebra [Z96]. Such a vertex algebra $V$ is called a chiralization 
of an algebra $A$ if $A(V) \cong A$. We claim that if $\mathfrak{g}$ belongs to the Deligne exceptional series outside the type $A$ and if $k=-h^{\vee} / 6-1$, then $V_{k}(\mathfrak{g})$ is a chiralization of $\mathbb{C} \oplus U(\mathfrak{g}) / \mathcal{J}_{0}$. Namely,

$$
A\left(V_{k}(\mathfrak{g})\right) \cong U(\mathfrak{g}) / \mathcal{J}_{\mathcal{W}} \cong \mathbb{C} \times U(\mathfrak{g}) / \mathcal{J}_{0},
$$

for some ideal $\mathcal{J}_{\mathcal{W}}$ (cf. Proposition 2.2 and Theorem 3.1). Hence the classification of simple highest weight $U(\mathfrak{g}) / \mathcal{J}_{0}$-modules obtained in [J98] gives the classification of simple highest weight $V_{k}(\mathfrak{g})$-modules thanks to Zhu's theorem [Z96], which for types $G_{2}, D_{4}, F_{4}$ reproves the earlier results obtained in [AL11] and [Pe07b, Pe13].

Another consequence of the fact $X_{V_{k}(\mathfrak{g})} \subset \mathcal{N}$ is that the $D$-module on the moduli stack of $G$-bundles on a curve obtained from $V_{k}(\mathfrak{g})$ by the Harish-Chandra localization [BD, FBZ04] has its micro-local support inside the global nilpotent cone. It would be very interesting to consider the associated modular functor (cf. [FM97]), or the corresponding conformal field theory (cf. [CR12, CR13]). We hope to come back to this point in our future work.

In physics literature the affine vertex algebras in Theorem 1.1 (1) have been studied in the work $\left[\mathrm{BLL}^{+} 15\right]$ of Beem, Lemos, Liendo, Peelaers, Rastelli and van Rees in connection with four dimensional superconformal field theory. The associated varieties of these vertex algebras seem to describe the Higgs branch of the corresponding four dimensional theory. We also hope to come back to this point in our future work.

Theorem 1.1, or its proof, has the following important application:

Let $\mathcal{W}^{k}\left(\mathfrak{g}, f_{\theta}\right)$ be the $W$-algebra associated with $\left(\mathfrak{g}, f_{\theta}\right)$ at level $k$ [KRW03], which is a conformal vertex algebra with central charge

$$
c(k)=\frac{k \operatorname{dim} \mathfrak{g}}{k+h^{\vee}}-6 k+h^{\vee}-4
$$

provided that $k \neq-h^{\vee}$. Note that if $\mathfrak{g}$ belongs to the Deligne exceptional series,

$$
c(k)=-\frac{6\left(k+h^{\vee} / 6+1\right)\left(\left(h^{\vee} / 6+1\right) k-\left(h^{\vee}-4\right) h^{\vee} / 6\right)}{\left(k+h^{\vee}\right)\left(h^{\vee} / 6+1\right)},
$$

so that $c(k)=0$ for $k=-h^{\vee} / 6-1$.

Denote by $\mathcal{W}_{k}\left(\mathfrak{g}, f_{\theta}\right)$ the unique simple quotient of $\mathcal{W}^{k}\left(\mathfrak{g}, f_{\theta}\right)$. Since $X_{\mathcal{W}^{k}\left(\mathfrak{g}, f_{\theta}\right)}$ is naturally isomorphic to the Slodowy slice $\mathcal{S}_{\text {min }}$ at $f_{\theta}$ ([DSK06, Ar15a]), with

$$
\mathcal{S}_{\text {min }}:=f_{\theta}+\mathfrak{g}^{e_{\theta}}, \quad \mathfrak{g}^{e_{\theta}}=\left\{x \in \mathfrak{g} \mid\left[x, e_{\theta}\right]=0\right\},
$$

the variety $X_{\mathcal{W}_{k}\left(\mathfrak{g}, f_{\theta}\right)}$ is a $\mathbb{C}^{*}$-invariant, Poisson subvariety of $\mathcal{S}_{\text {min }}$.

It is known [DSK06] that the (Ramond twisted) Zhu's algebra of $\mathcal{W}^{k}\left(\mathfrak{g}, f_{\theta}\right)$ is naturally isomorphic to the finite $W$-algebra $U\left(\mathfrak{g}, f_{\theta}\right)$ associated with $\left(\mathfrak{g}, f_{\theta}\right)$ introduced by Premet [Pr02].

Premet [Pr07] has shown that the Joseph ideal is closely connected with onedimensional representations of $U\left(\mathfrak{g}, f_{\theta}\right)$. The chiralization of $U(\mathfrak{g}) / \mathcal{J}_{W}$ explained above is closely related with one-dimensional representations of $\mathcal{W}^{k}\left(\mathfrak{g}, f_{\theta}\right)$ as well. The significant difference in the affine setting is that $\mathcal{W}^{k}\left(\mathfrak{g}, f_{\theta}\right)$ does not necessarily admit one-dimensional representations. In fact $\mathcal{W}^{k}\left(\mathfrak{g}, f_{\theta}\right), \mathfrak{g} \neq \mathfrak{s l}_{2}$, admits onedimensional representations if and only if $\mathcal{W}_{k}\left(\mathfrak{g}, f_{\theta}\right)=\mathbb{C}$, and this happens if and 
only if $\mathfrak{g}$ belongs to the Deligne exceptional series and $k=-h^{\vee} / 6-1$, or $\mathfrak{g}$ is of type $C_{l}$ and $k=-1 / 2$ (cf. Theorem 7.2).

Note that the trivial vertex algebra $\mathbb{C}$ is certainly a lisse vertex algebra. Here, recall that a vertex algebra $V$ is called lisse, or $C_{2}$-cofinite, if $\operatorname{dim} X_{V}=0$. Lisse vertex algebras may be regarded as an analogue of finite-dimensional algebras. One of remarkable properties of a lisse vertex algebra $V$ is the modular invariance of characters of modules [Z96, M04]. Further, if it is non-trivial and also rational, it is known [H08] that under some mild assumptions the category of $V$-modules forms a modular tensor category, which for instance yields an invariant of 3-manifolds, see [BK01].

In [Ar15a], in order to approach the Kac-Wakimoto conjecture [KW08] on the rationality of exceptional $W$-algebras, the first named author showed that each admissible affine vertex algebra produces exactly one lisse simple $W$-algebra. More precisely, the associated variety of an admissible affine vertex algebra $V_{k}(\mathfrak{g})$ is isomorphic to $\overline{\mathbb{O}}$ for some nilpotent orbit $\mathbb{O}$ of $\mathfrak{g}$, and if we take the nilpotent element $f$ from this orbit $\mathbb{O}$, then $\mathcal{W}_{k}(\mathfrak{g}, f)$ is lisse. Until very recently it has been widely believed that these $W$-algebras are all the lisse $W$-algebras, cf. [KW08]. However, it turned out that there are a lot more.

Theorem 1.2. (1) Let $\mathfrak{g}$ be of type $D_{4}, E_{6}, E_{7}, E_{8}$. For any integer $k$ that is equal to or greater than $-h^{\vee} / 6-1$, the simple $W$-algebra $\mathcal{W}_{k}\left(\mathfrak{g}, f_{\theta}\right)$ is lisse.

(2) Let $\mathfrak{g}$ be of type $D_{l}$ with $l \geqslant 5$. For any integer $k$ that is equal to or greater than-2, the simple $W$-algebra $\mathcal{W}_{k}\left(\mathfrak{g}, f_{\theta}\right)$ is lisse.

In the case that $k=-h^{\vee} / 6$, the first statement of Theorem 1.2 is a recent result of Kawasetsu [K15]. Kawasetsu actually proved that $\mathcal{W}_{-h^{\vee} / 6}\left(\mathfrak{g}, f_{\theta}\right)$ is rational and $C_{2}$-cofinite if $\mathfrak{g}$ belongs to the Deligne exceptional series, providing a first (surprising) example of rational and $C_{2}$-cofinite $W$-algebras that are not coming from admissible representations of $\widehat{\mathfrak{g}}$. Our present work is motivated by his result. It would be very interesting to know whether the lisse $W$-algebras appearing in Theorem 1.2 are rational or not. We hope to come back to this point in future work.

Acknowledgments. A part of this work was done while the first named author was staying at the Université de Poitiers in October, 2014 and at the Centro di Ricerca Matematica Ennio De Giorg in Pisa in December, 2014 and in January, 2015. He would like to thank both institutes. He would also like to thank Kazuya Kawasetsu for useful discussions, and Dražen Adamović for bringing the article [Pe13] to his attention. After submitting the first version of the present paper he had stimulating discussions with Leonardo Rastelli, Hiraku Nakajima, Takahiro Nishinaka and Yuji Tachikawa. He would like to thank all of them. His research is supported by JSPS KAKENHI Grant Numbers 25287004 and 26610006.

The second named author would like to thank Rupert Wei Tze Yu for bringing the article [GS04] to her attention, and Pierre Torasso for useful discussions about central characters. Her research is supported by the ANR Project GERCHER Grant number ANR-2010-BLAN-110-02. 
Both authors thank Dražen Adamović, Victor Kac, Ozren Perše and Weiqiang Wang for their comments on the first version of this article.

\section{Minimal nilpotent orbit Closures and Joseph ideals}

Let $J_{0}$ be the prime ideal of $S(\mathfrak{g})$ corresponding to the minimal nilpotent orbit closure $\overline{\mathbb{O}_{\text {min }}}$ in $\mathfrak{g}^{*}$.

Suppose that $\mathfrak{g}$ is not of type $A$. According to Kostant, $J_{0}$ is generated by a $\mathfrak{g}$-submodule $L_{\mathfrak{g}}(0) \oplus W$ in $S^{2}(\mathfrak{g})$, such that

$$
S^{2}(\mathfrak{g})=L_{\mathfrak{g}}(2 \theta) \oplus L_{\mathfrak{g}}(0) \oplus W,
$$

where $L_{\mathfrak{g}}(\lambda)$ is the irreducible representation of $\mathfrak{g}$ with highest weight $\lambda$ and $\theta$ is the highest root of $\mathfrak{g}$.

Note that the above decomposition of $S^{2}(\mathfrak{g})$ still holds in type $A$, [G82, Chapter IV, Proposition 2],

Also, note that $L_{\mathfrak{g}}(0)=\mathbb{C} \Omega$ where $\Omega$ is the Casimir element in $S(\mathfrak{g})$.

Lemma 2.1. Suppose that $\mathfrak{g}$ is not of type A. The ideal $J_{W}$ in $S(\mathfrak{g})$ generated by $W$ contains $\Omega^{2}$, and hence, $\sqrt{J_{W}}=J_{0}$.

Proof. By the proof of [GS04, Theorem 3.1] $J_{W}$ contains $\mathfrak{g} \cdot \Omega$, and the assertion follows.

The structure of $W$ was determined by Garfinkle [G82]. Set

$$
\mathfrak{g}(j)=\left\{x \in \mathfrak{g} \mid\left[h_{\theta}, x\right]=2 j x\right\} .
$$

Then

$$
\begin{aligned}
\mathfrak{g}=\mathfrak{g}(-1) \oplus \mathfrak{g}(-1 / 2) \oplus \mathfrak{g}(0) \oplus \mathfrak{g}(1 / 2) \oplus \mathfrak{g}(1), \\
\mathfrak{g}(-1)=\mathbb{C} f_{\theta}, \mathfrak{g}(1)=\mathbb{C} e_{\theta}, \mathfrak{g}(0)=\mathbb{C} h_{\theta} \oplus \mathfrak{g}^{\natural}, \mathfrak{g}^{\natural}=\left\{x \in \mathfrak{g}(0) \mid\left(h_{\theta} \mid x\right)=0\right\} .
\end{aligned}
$$

The subalgebra $\mathfrak{g}^{\natural}$ is a reductive subalgebra of $\mathfrak{g}$ whose simple roots are the simple roots of $\mathfrak{g}$ perpendicular to $\theta$. Write

$$
\left[\mathfrak{g}^{\natural}, \mathfrak{g}^{\natural}\right]=\bigoplus_{i \geqslant 1} \mathfrak{g}_{i}
$$

as a direct sum of simple summands, and let $\theta_{i}$ be the highest root of $\mathfrak{g}_{i}$.

If $\mathfrak{g}$ is neither of type $A_{l}$ nor $C_{l}$,

$$
W=\bigoplus_{i \geqslant 1} L_{\mathfrak{g}}\left(\theta+\theta_{i}\right)
$$

If $\mathfrak{g}$ is of type $C_{l}$, then $\mathfrak{g}^{\natural}$ is simple of type $C_{l-1}$, so that there is a unique $\theta_{1}$, and we have

$$
W=L_{\mathfrak{g}}\left(\theta+\theta_{1}\right) \oplus L_{\mathfrak{g}}\left(\frac{1}{2}\left(\theta+\theta_{1}\right)\right) .
$$

If $\mathfrak{g}$ is not of type $A$, it is known [J76, GS04] that there exists a unique completely prime ideal $\mathcal{J}_{0}$ in $U(\mathfrak{g})$, called the Joseph ideal, whose associated variety is $\overline{\mathbb{O}_{\text {min }}}$. It is known that $\mathcal{J}_{0}$ is maximal and primitive. By [G82, GS04] $\mathcal{J}_{0}$ is generated 
by $W$ and $\Omega-c_{0}$, where $W$ is identified with a $\mathfrak{g}$-submodule of $U(\mathfrak{g})$ by the $\mathfrak{g}$ module isomorphism $S(\mathfrak{g}) \cong U(\mathfrak{g})$ and $c_{0}$ is the eigenvalue of $\Omega$ for the infinitesimal character that Joseph obtained in [J76, Table p.15]. We have

$$
\operatorname{gr} \mathcal{J}_{0}=J_{0}=\sqrt{J_{W}}
$$

and this shows that $\mathcal{J}_{0}$ is indeed completely prime.

Let $\mathcal{J}_{W}$ be the two-sided ideal of $U(\mathfrak{g})$ generated by $W$.

Proposition 2.2. We have an algebra isomorphism

$$
U(\mathfrak{g}) / \mathcal{J}_{W} \cong \mathbb{C} \times U(\mathfrak{g}) / \mathcal{J}_{0} .
$$

Proof. By the proof of [GS04, Theorem 3.1], $\mathcal{J}_{W}$ contains $\left(\Omega-c_{0}\right) \mathfrak{g}$. Hence it contains $\left(\Omega-c_{0}\right) \Omega$. Since $c_{0} \neq 0$, we have an isomorphism of algebras

$$
U(\mathfrak{g}) / \mathcal{J}_{W} \stackrel{\sim}{\rightarrow} U(\mathfrak{g}) /\left\langle\mathcal{J}_{W}, \Omega\right\rangle \times U(\mathfrak{g}) /\left\langle\mathcal{J}_{W}, \Omega-c_{0}\right\rangle .
$$

As we have explained above, $\left\langle\mathcal{J}_{W}, \Omega-c_{0}\right\rangle=\mathcal{J}_{0}$. Also, since $\mathcal{J}_{W}$ contains $\left(\Omega-c_{0}\right) \mathfrak{g}$, $\left\langle\mathcal{J}_{W}, \Omega\right\rangle$ contains $\mathfrak{g}$. Therefore $U(\mathfrak{g}) /\left\langle\mathcal{J}_{W}, \Omega\right\rangle=\mathbb{C}$ as required.

\section{A Lifting of Joseph ideals}

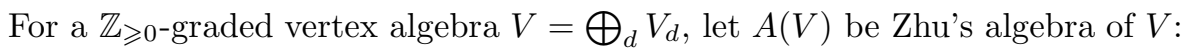

$$
A(V)=V / V \circ V
$$

where $V \circ V$ is the $\mathbb{C}$-span of the vectors

$$
a \circ b:=\sum_{i \geqslant 0}\left(\begin{array}{c}
\Delta \\
i
\end{array}\right) a_{(i-2)} b
$$

for $a \in V_{\Delta}, \Delta \in \mathbb{Z}_{\geqslant 0}, b \in V$, and $V \rightarrow($ End $V)\left[\left[z, z^{-1}\right]\right], a \mapsto \sum_{n \in \mathbb{Z}} a_{(n)} z^{-n-1}$, denotes the state-field correspondence. The space $A(V)$ is a unital associative algebra with respect to the multiplication defined by

$$
a * b:=\sum_{i \geqslant 0}\left(\begin{array}{c}
\Delta \\
i
\end{array}\right) a_{(i-1)} b
$$

for $a \in V_{\Delta}, \Delta \in \mathbb{Z}_{\geqslant 0}, b \in V$. More generally, for a $V$-module $M$, a bimodule $A(M)$ over $A(V)$ is defined similarly ([FZ92]).

Zhu's algebra $A(V)$ naturally acts on the top degree component $M_{t o p}$ of a $\mathbb{Z}_{\geqslant 0^{-}}$ graded $V$-module $M$, and $M \mapsto M_{t o p}$ gives [Z96] a one-to-one correspondence between simple graded $V$-modules and simple $A(V)$-modules.

The vertex algebra $V$ is called a chiralization of an algebra $A$ if $A(V) \cong A$.

For instance, consider the universal affine vertex algebra $V^{k}(\mathfrak{g})$. A $V^{k}(\mathfrak{g})$-module is the same as a smooth $\widehat{\mathfrak{g}}^{\prime}$-module of level $k$, where $\widehat{\mathfrak{g}}^{\prime}=[\widehat{\mathfrak{g}}, \widehat{\mathfrak{g}}]=\mathfrak{g}\left[t, t^{-1}\right] \oplus \mathbb{C} K$. Zhu's algebra $A\left(V^{k}(\mathfrak{g})\right.$ ) is naturally isomorphic to $U(\mathfrak{g})$ ([FZ92], see also [Ar16, Lemma 2.3]), and hence, $V^{k}(\mathfrak{g})$ is a chiralization of $U(\mathfrak{g})$. The top degree component of the irreducible highest weight representation $L(\lambda)$ of $\widehat{\mathfrak{g}}$ with highest weight $\lambda$ is $L_{\mathfrak{g}}(\bar{\lambda})$, where $\bar{\lambda}$ is the restriction of $\lambda$ to the Cartan subalgebra of $\mathfrak{g}$. 
Let $\widehat{\mathcal{J}}_{k}$ be the unique maximal ideal of $V^{k}(\mathfrak{g})$, so that

$$
V_{k}(\mathfrak{g})=V^{k}(\mathfrak{g}) / \widehat{\mathcal{J}}_{k}
$$

We have the exact sequence $A\left(\widehat{\mathcal{J}}_{k}\right) \rightarrow U(\mathfrak{g}) \rightarrow A\left(V_{k}(\mathfrak{g})\right) \rightarrow 0$ since the functor $A(?)$ is right exact and thus $A\left(V_{k}(\mathfrak{g})\right)$ is the quotient of $U(\mathfrak{g})$ by the the image $\mathcal{I}_{k}$ of $A\left(\widehat{\mathcal{J}}_{k}\right)$ in $U(\mathfrak{g})$ :

$$
A\left(V_{k}(\mathfrak{g})\right)=U(\mathfrak{g}) / \mathcal{I}_{k} .
$$

One may ask whether $\mathcal{I}_{k}$ coincides with the Joseph ideal $\mathcal{J}_{0}$ for some $k \in \mathbb{C}$, so that $V_{k}(\mathfrak{g})$ is a chiralization of $U(\mathfrak{g}) / \mathcal{J}_{0}$. But this can never happen. Indeed, $U(\mathfrak{g}) / \mathcal{J}_{0}$ does not admit finite dimensional representations while $\mathbb{C}$ is always an $A\left(V_{k}(\mathfrak{g})\right)$-module as $V_{k}(\mathfrak{g})$ is a module over itself and $V_{k}(\mathfrak{g})_{\text {top }}=\mathbb{C}$. However, by Proposition 2.2, it makes sense to ask the same question for the ideal $\mathcal{J}_{W}$.

Theorem 3.1. Assume that $\mathfrak{g}$ belongs to the Deligne exceptional series outside the type $A$ and that $k=-h^{\vee} / 6-1$. Then $V_{k}(\mathfrak{g})$ is a chiralization of $U(\mathfrak{g}) / \mathcal{J}_{\mathcal{W}}$, that is,

$$
A\left(V_{k}(\mathfrak{g})\right) \cong U(\mathfrak{g}) / \mathcal{J}_{\mathcal{W}} \cong \mathbb{C} \times U(\mathfrak{g}) / \mathcal{J}_{0} .
$$

In particular, since $\mathcal{J}_{0}$ is maximal, the irreducible highest weight representation $L(\lambda)$ of $\widehat{\mathfrak{g}}$ is a $V_{k}(\mathfrak{g})$-module if and only if

$$
\bar{\lambda}=0 \quad \text { or } \quad \operatorname{Ann}_{U(\mathfrak{g})} L_{\mathfrak{g}}(\bar{\lambda})=\mathcal{J}_{0} .
$$

According to $[\mathrm{J} 98,4.3]$, the weights $\mu$ such that $\operatorname{Ann}_{U(\mathfrak{g})} L_{\mathfrak{g}}(\mu)=\mathcal{J}_{0}$ are

$$
w \circ\left(\lambda_{0}-\rho\right):=w\left(\lambda_{0}\right)-\rho, \quad w \in \mathrm{W}_{0},
$$

where the weight $\lambda_{0}$ and the subset $\mathrm{W}_{0}$ of the Weyl group $\mathrm{W}$ of $\mathfrak{g}$ are described in Table 1. Here we adopt the standard Bourbaki numbering for the simple roots $\left\{\alpha_{1}, \ldots, \alpha_{1}\right\}$ of $\mathfrak{g}$, and we denote by $\varpi_{1}, \ldots, \varpi_{l}$ the corresponding fundamental weights.

\begin{tabular}{|c|c|c|c|}
\hline & $-\frac{h^{\vee}}{6}-1$ & $\lambda_{0}$ & $\mathrm{~W}_{0}$ \\
\hline$G_{2}$ & $-\frac{5}{3}$ & $\varpi_{1}+\frac{1}{3} \varpi_{2}$ & $\left\{1, s_{2}\right\}$ \\
\hline$D_{4}$ & -2 & $\varpi_{1}+\varpi_{3}+\varpi_{4}$ & $\left\{1, s_{1}, s_{3}, s_{4}\right\}$ \\
\hline$F_{4}$ & $-\frac{5}{2}$ & $\frac{1}{2} \varpi_{1}+\frac{1}{2} \varpi_{2}+\varpi_{3}+\varpi_{4}$ & $\left\{1, s_{1}, s_{2}\right\}$ \\
\hline$E_{6}$ & -3 & $\varpi_{1}+\varpi_{2}+\varpi_{3}+\varpi_{5}+\varpi_{6}$ & $\left\{1, s_{2}, s_{3}, s_{1} s_{3}, s_{5}, s_{6} s_{5}\right\}$ \\
\hline$E_{7}$ & -4 & $\varpi_{1}+\varpi_{2}+\varpi_{3}+\varpi_{5}+\varpi_{6}+\varpi_{7}$ & $\left\{1, s_{2}, s_{3}, s_{1} s_{3}, s_{5}, s_{6} s_{5}, s_{7} s_{6} s_{5}\right\}$ \\
\hline$E_{8}$ & -6 & $\varpi_{1}+\varpi_{2}+\varpi_{3}+\varpi_{5}+\varpi_{6}+\varpi_{7}+\varpi_{8}$ & $\left\{1, s_{2}, s_{3}, s_{1} s_{3}, s_{5}, s_{6} s_{5}, s_{7} s_{6} s_{5}, s_{8} s_{7} s_{6} s_{5}\right\}$ \\
\hline
\end{tabular}

TABLE $1 .-h^{\vee} / 6-1, \lambda_{0}$ and $\mathrm{W}_{0}$

Note that the last statement of Theorem 3.1 reproves the earlier results [AL11, Proposition 3.6 (1)] for type $G_{2}$, [Pe13, Theorem 4.3] for type $D_{4}$ and [Pe07b, Theorem 6.4] for type $F_{4}$. 
For types $G_{2}$ and $F_{4}$, the level $k=-h^{\vee} / 6-1$ is admissible, that is, $k \Lambda_{0}$ is an admissible weight [KW89] for $\widehat{\mathfrak{g}}$. Using [Ar16, Proposition 3.3] one finds that

$$
\left\{k \Lambda_{0}, w \circ\left(\lambda_{0}-\rho\right)+k \Lambda_{0} \mid w \in \mathrm{W}_{0}\right\}
$$

is exactly the set of admissible weights of level $k$ whose integral Weyl group is isomorphic to that of $k \Lambda_{0}$, which agrees with [Ar16, Main Theorem].

Theorem 3.1 will be proven at the end of Section 4 .

\section{Singular vectors of AfFine Vertex Algebra of Degree 2}

By the PBW theorem, we have $V^{k}(\mathfrak{g}) \cong U\left(\mathfrak{g}\left[t^{-1}\right] t^{-1}\right)$ as $\mathbb{C}$-vector spaces. Below we often identify $V^{k}(\mathfrak{g})$ with $U\left(\mathfrak{g}\left[t^{-1}\right] t^{-1}\right)$.

The vertex algebra $V^{k}(\mathfrak{g})$ is naturally graded:

$$
V^{k}(\mathfrak{g})=\bigoplus_{d \in \mathbb{Z}} V^{k}(\mathfrak{g})_{d}, \quad V^{k}(\mathfrak{g})_{d}=\left\{v \in V^{k}(\mathfrak{g}) \mid D v=-d v\right\}
$$

Note that each homogeneous component $V^{k}(\mathfrak{g})_{d}$ is a finite-dimensional $\mathfrak{g}$-submodule of $V^{k}(\mathfrak{g})$.

Lemma 4.1. We have a $\mathfrak{g}$-module embedding

$$
\sigma_{d}: S^{d}(\mathfrak{g}) \hookrightarrow V^{k}(\mathfrak{g})_{d}, \quad x_{1} \ldots x_{d} \mapsto \frac{1}{d !} \sum_{\sigma \in \mathfrak{S}_{d}} x_{\sigma(1)}(-1) \ldots x_{\sigma(d)}(-1) .
$$

Let $v$ be a singular vector in $S^{d}(\mathfrak{g})$. Then $\sigma_{d}(v)$ is a singular vector of $V^{k}(\mathfrak{g})$ if and only if $f_{\theta}(1) \sigma_{d}(v)=0$. For $d=2$, we will simply denote by $\sigma$ the embedding $\sigma_{d}$

Let $W=\bigoplus_{i} W_{i}$ be the decomposition of $W$ into irreducible submodules, and let $w_{i}$ be a highest weight vector of $W_{i}$.

Theorem 4.2. (1) Assume that $\mathfrak{g}$ belongs to the Deligne exceptional series outside the type $A$.

(a) For any $i, \sigma\left(w_{i}\right)$ is a singular vector of $V^{k}(\mathfrak{g})$ if and only if

$$
k=-h^{\vee} / 6-1 .
$$

(b) Assume that $\mathfrak{g}$ is not of type $G_{2}$. For each $n \in \mathbb{Z}_{\geqslant 0}$ and each $i$, $\sigma\left(w_{i}\right)^{n+1}$ is a singular vector of $V^{k}(\mathfrak{g})$ if and only if

$$
k=n-h^{\vee} / 6-1 .
$$

(2) Let $\mathfrak{g}$ be of type $B_{l}, l \geqslant 3$, so that $W=W_{1} \oplus W_{2}$ where $W_{1} \cong L_{\mathfrak{g}}\left(\theta+\theta_{1}\right)=$ $L_{\mathfrak{g}}\left(2 \varpi_{1}\right)$ and $W_{2} \cong L_{\mathfrak{g}}\left(\theta+\theta_{2}\right)=L_{\mathfrak{g}}\left(\varpi_{4}\right)$ if $l \geqslant 5$ (and $W_{2} \cong L_{\mathfrak{g}}\left(\theta+\theta_{2}\right)=$ $L_{\mathfrak{g}}\left(2 \varpi_{l}\right)$ if $\left.l=3,4\right)$.

(a) ([Pe07a]) For each $n \in \mathbb{Z}_{\geqslant 0}, \sigma\left(w_{1}\right)^{n+1}$ is a singular vector of $V^{k}(\mathfrak{g})$ if and only if

$$
k=n-l+3 / 2 .
$$


(b) For each $n \in \mathbb{Z}_{\geqslant 0}, \sigma\left(w_{2}\right)^{n+1}$ is a singular vector of $V^{k}(\mathfrak{g})$ if and only if

$$
k=n-2 .
$$

(3) ([Ad94]) Let $\mathfrak{g}$ be of type $C_{l}, l \geqslant 2$, so that $W=W_{1} \oplus W_{2}$ where $W_{1} \cong$ $L_{\mathfrak{g}}\left(\theta+\theta_{1}\right)=L_{\mathfrak{g}}\left(2 \varpi_{2}\right)$ and $W_{2} \cong L_{\mathfrak{g}}\left(\frac{1}{2} \theta+\theta_{1}\right)=L_{\mathfrak{g}}\left(\varpi_{2}\right)$. For each $n \in \mathbb{Z}_{\geqslant 0}$, $\sigma\left(w_{1}\right)^{n+1}$ is a singular vector of $V^{k}(\mathfrak{g})$ if and only if

$$
k=n-1 / 2 \text {. }
$$

(4) Let $\mathfrak{g}$ of type $D_{l}, l \geqslant 5$, so that $W=W_{1} \oplus W_{2}$ where $W_{1} \cong L_{\mathfrak{g}}\left(\theta+\theta_{1}\right)=$ $L_{\mathfrak{g}}\left(2 \varpi_{1}\right)$ and $W_{2} \cong L_{\mathfrak{g}}\left(\theta+\theta_{2}\right)=L_{\mathfrak{g}}\left(\varpi_{4}\right)$ if $l \geqslant 6$ (and $W_{2} \cong L_{\mathfrak{g}}\left(\theta+\theta_{2}\right)=$ $L_{\mathfrak{g}}\left(\varpi_{4}+\varpi_{5}\right)$ if $\left.l=5\right)$.

(a) ([Pe13]) For each $n \in \mathbb{Z}_{\geqslant 0}, \sigma\left(w_{1}\right)^{n+1}$ is a singular vector of $V^{k}(\mathfrak{g})$ if and only if

$$
k=n-l+2 .
$$

(b) For each $n \in \mathbb{Z}_{\geqslant 0}, \sigma\left(w_{2}\right)^{n+1}$ is a singular vector of $V^{k}(\mathfrak{g})$ if and only if

$$
k=n-2 .
$$

Note that (1) for $D_{4}$ is also a particular case of [Pe13], that (1) (a) for $G_{2}$ was proved in [AL11], and that (1) for $F_{4}$ was proved in [Pe07b].

Proof. (1) Assume that $\mathfrak{g}$ is of type $D_{4}, E_{6}, E_{7}, E_{8}$. Then it is enough to prove (b).

For $E_{6}, E_{7}, E_{8}, W=W_{1}$. For $D_{4}, W=W_{1} \oplus W_{2} \oplus W_{3}$. Using the Dynkin automorphism, we can assume that $i=1$, and that $W_{1}=L_{\mathfrak{g}}\left(2 \varpi_{1}\right)$.

For types $E_{6}$ and $E_{7}, \mathfrak{g}$ is of depth one, [G82, Chapter IV, Definition 1], and $\left(\theta-\theta_{1}\right) / 2$ is not a root.

Then we apply [G82, Chapter IV, Proposition 11] to construct a singular vector $w_{1}$ for $W_{1}$. Table 2 describes the pairs of positives roots $\left(\beta_{j}, \delta_{j}\right)$ such that

$$
\beta_{j}+\delta_{j}=\theta-\theta_{1} .
$$

The number of such pairs turns out to be equal to $h^{\vee} / 6+1$. In this table, a positive root $\gamma$ is represented by $\left(k_{1}, \ldots, k_{l}\right)$ if $\gamma=\sum_{j=1}^{l} k_{j} \alpha_{j}$.

Choose a Chevalley basis $\left\{h_{i}\right\}_{i} \cup\left\{e_{\alpha}, f_{\alpha}\right\}_{\alpha}$ of $\mathfrak{g}$ so that the conditions of [G82, Chapter IV, Definition 6] are fulfilled, that is

$$
\forall j, \quad\left[e_{\delta_{j}},\left[e_{\beta_{j}}, e_{\theta_{1}}\right]\right]=e_{\theta}, \quad\left[e_{\beta_{j}}, e_{\theta_{1}}\right]=e_{\beta_{j}+\theta_{1}}, \quad\left[e_{\delta_{j}}, e_{\theta_{1}}\right]=e_{\delta_{j}+\theta_{1}} .
$$

Then set

$$
w_{1}:=e_{\theta} e_{\theta_{1}}-\sum_{k=1}^{\frac{h^{\vee}}{6}+1} e_{\beta_{j}+\theta_{1}} e_{\delta_{j}+\theta_{1}},
$$


so that

$$
\begin{aligned}
\sigma\left(w_{1}\right)= & \frac{1}{2}\left(e_{\theta}(-1) e_{\theta_{1}}(-1)+e_{\theta_{1}}(-1) e_{\theta}(-1)\right. \\
& \left.\quad-\sum_{k=1}^{\frac{h^{\vee}}{6}+1}\left(e_{\beta_{j}+\theta_{1}}(-1) e_{\delta_{j}+\theta_{1}}(-1)+e_{\delta_{j}+\theta_{1}}(-1) e_{\beta_{j}+\theta_{1}}(-1)\right)\right) .
\end{aligned}
$$

We observe using the relations (1) that for each $j$,

$$
\left[\left[f_{\theta}, e_{\beta_{j}+\theta_{1}}\right], e_{\delta_{j}+\theta_{1}}\right]=\left[\left[f_{\theta}, e_{\delta_{j}+\theta_{1}}\right], e_{\beta_{j}+\theta_{1}}\right]=-e_{\theta_{1}} .
$$

By (2), we get:

$$
\begin{aligned}
f_{\theta}(1) \cdot \sigma\left(w_{1}\right)= & \left(\left[f_{\theta}, e_{\theta}\right](0)+k+\frac{h^{\vee}}{6}+1\right) e_{\theta_{1}}(-1) \\
& -\sum_{k=1}^{\frac{h^{\vee}}{6}+1}\left(e_{\beta_{j}+\theta_{1}}(-1)\left[f_{\theta}, e_{\delta_{j}+\theta_{1}}\right](0)+e_{\delta_{j}+\theta_{1}}(-1)\left[f_{\theta}, e_{\beta_{j}+\theta_{1}}\right](0)\right) .
\end{aligned}
$$

Observe that

$$
\left[f_{\theta}, e_{\theta}\right](0) \cdot \sigma\left(w_{1}\right)=-2 \sigma\left(w_{1}\right)
$$

since $\left\langle\theta+\theta_{1}, \theta^{\vee}\right\rangle=\left\langle\theta, \theta^{\vee}\right\rangle=2$, and that

$$
\left[f_{\theta}, e_{\delta_{j}+\theta_{1}}\right](0) \cdot \sigma\left(w_{1}\right)=\left[f_{\theta}, e_{\beta_{j}+\theta_{1}}\right](0) \cdot \sigma\left(w_{1}\right)=0
$$

since $-\theta+\delta_{j}+\theta_{1},-\theta+\beta_{j}+\theta_{1}$ are perpendicular to $\theta+\theta_{1}$, the weight of $\sigma\left(w_{1}\right)$, for each $j$. In addition, since $\beta_{j}+2 \theta_{1}, \delta_{j}+2 \theta_{1}$ are not roots, $\left[e_{\theta_{1}}(-1), \sigma\left(w_{1}\right)\right]=0$. So, for any $n \in \mathbb{Z}_{\geqslant 0}$ we get,

$$
\begin{aligned}
& f_{\theta}(1) \cdot \sigma\left(w_{1}\right)^{n+1} \\
& =\sigma\left(w_{1}\right)^{n}\left(k+\frac{h^{\vee}}{6}+1\right) e_{\theta_{1}}(-1)+\sum_{j=1}^{n}\left(\sigma\left(w_{1}\right)^{n-j}\left(\left[f_{\theta}, e_{\theta}\right](0)+k+\frac{h^{\vee}}{6}+1\right) \cdot \sigma\left(w_{1}\right)^{j} e_{\theta_{1}}(-1)\right) \\
& =\sum_{j=0}^{n}\left(-2 j+k+\frac{h^{\vee}}{6}+1\right) \sigma\left(w_{1}\right)^{n} e_{\theta_{1}}(-1)=(n+1)\left(-n+k+\frac{h^{\vee}}{6}+1\right) \sigma\left(w_{1}\right)^{n} e_{\theta_{1}}(-1) .
\end{aligned}
$$

Hence $\sigma\left(w_{1}\right)^{n+1}$ is a singular vector of $V^{k}(\mathfrak{g})$ for $k=n-h^{\vee} / 6-1$.

Assume that $\mathfrak{g}$ has type $E_{8}$. Then $\mathfrak{g}$ is not of depth one and we follow the construction of [G82, Chapter IV, §4]. According to [G82, Chapter IV, §4], there is a positive root $\alpha$ such that the algebra $\tilde{\mathfrak{g}}$ generated by $e_{\alpha}, e_{2}, \ldots, e_{8}, f_{\alpha}, f_{2}, \ldots, f_{8}$ has type $D_{8}$, where $e_{i}, f_{i}, i=1, \ldots, 8$ are the generators of a Chevalley basis of $\mathfrak{g}$ corresponding to the simple roots $\alpha_{1}, \ldots, \alpha_{8}$ in the Bourbaki numbering. Moreover, we have that $\alpha=\theta_{1}$. Then we apply the construction of [G82, Chapter IV, $\left.\S 1\right]$ to the algebra $\tilde{\mathfrak{g}}$ which is of depth one. One can choose our Chevalley basis $\left\{h_{i}\right\}_{i} \cup$ $\left\{e_{\alpha}, f_{\alpha}\right\}_{\alpha}$ of $\mathfrak{g}$ so that the conditions of [G82, Chapter IV, Definition 6] are fulfilled for $\tilde{\mathfrak{g}}$. Note that the highest root of $\tilde{\mathfrak{g}}$ is $\theta$, that is, the same as for $\mathfrak{g}$.

Then we apply as in cases $E_{6}, E_{7}$ the construction of [G82, Chapter IV, Proposition 11]. Table 2 describes the pairs of positives roots $\left(\beta_{j}, \delta_{j}\right)$ such that

$$
\beta_{j}+\delta_{j}=\theta-\theta_{1} .
$$

The number of such pairs is $h^{\vee} / 6+1$ too. 
Then we set

$$
w_{1}:=e_{\theta} e_{\theta_{1}}-\sum_{k=1}^{\frac{h^{\vee}}{6}+1} e_{\beta_{j}+\theta_{1}} e_{\delta_{j}+\theta_{1}} .
$$

We verify as for the types $E_{6}, E_{7}$ that $\sigma\left(w_{1}\right)^{n+1}$ is a singular vector of $V^{k}(\mathfrak{g})$ for $k=n-h^{\vee} / 6-1$.

\begin{tabular}{|c|c|c|c|c|}
\hline Type & $D_{4}$ & $E_{6}$ & $E_{7}$ & $E_{8}$ \\
\hline$h^{\vee} / 6+1$ & 2 & 3 & 4 & 6 \\
\hline$\theta$ & $(1211)$ & $(122321)$ & $(2234321)$ & $(23465432)$ \\
\hline$\theta_{1}$ & $(1000)$ & $(101111)$ & $(0112221)$ & $(22343210)$ \\
\hline$\left(\beta_{j}, \delta_{j}\right)$, & $(0100),(0111)$ & $(010000),(011210)$ & $(1000000),(1122100)$ & $(00000001),(01122221)$ \\
$\beta_{j}+\delta_{j}=\theta-\theta_{1}$ & $(0101),(0110)$ & $(010100),(011110)$ & $(1010000),(1112100)$ & $(00000011),(01122211)$ \\
& & $(010110),(010100)$ & $(1011000),(1111100)$ & $(00000111),(01122111)$ \\
& & & & $(00001111),(01121111)$ \\
& & & $(00011111),(01111111)$ \\
& & & & $(01011111),(00111111)$ \\
\hline
\end{tabular}

TABle 2. Data for $D_{4}, E_{6}, E_{7}, E_{8}$

(2) (b) and (4) (b) Assume that $\mathfrak{g}$ is of type $B_{l}, l \geqslant 3$, or of type $D_{l}, l \geqslant 5$. Then in both cases, $\theta_{2}$ is the highest root of the root system generated by $\alpha_{3}, \ldots, \alpha_{l}$, $\left(\theta-\theta_{2}\right) / 2$ is not a root and there are precisely two pairs $\left(\beta_{j}, \delta_{j}\right)$ such that $\beta_{j}+\delta_{j}=$ $\theta-\theta_{2}$. Namely, these pairs are:

$$
\left(\beta_{1}, \delta_{1}\right)=\left(\alpha_{2}, \alpha_{1}+\alpha_{2}+\alpha_{3}\right) \quad \text { and } \quad\left(\beta_{2}, \delta_{2}\right)=\left(\alpha_{2}+\alpha_{3}, \alpha_{1}+\alpha_{2}\right) .
$$

According to [G82, Chapter IV,Proposition 11],

$$
w_{2}:=e_{\theta} e_{\theta_{2}}-\sum_{k=1}^{2} e_{\beta_{j}+\theta_{2}} e_{\delta_{j}+\theta_{2}}
$$

is a singular vector for $\mathfrak{g}$. Moreover, all bracket relations (1) and (2) hold as in case $(1)^{1}$, with $\theta_{2}$ in place of $\theta_{1}$. Hence we get,

$$
f_{\theta}(1) \cdot \sigma\left(w_{2}\right)^{n+1}=(-n+k+2) \sigma\left(w_{2}\right)^{n} e_{\theta_{2}}(-1) .
$$

The statement follows.

Remark 4.3. If $\mathfrak{g}$ is of type $C_{l}, l \geqslant 3$, we can construct a singular vector for $V^{k}(\mathfrak{g})$ of weight $\frac{1}{2}\left(\theta+\theta_{1}\right)$ with $k=-(l+6) / 2$ as follows.

Set

$$
\theta_{0}:=\left(\theta+\theta_{1}\right) / 2=\alpha_{1}+2\left(\alpha_{2}+\cdots+\alpha_{l-1}\right)+\alpha_{l} .
$$

For $j \in\{2, \ldots, l\}$, set

$$
\beta_{j}:=\alpha_{1}+\alpha_{2}+\cdots+\alpha_{j-1}, \quad \delta_{j}:=\alpha_{2}+\cdots+\alpha_{j-1}+2\left(\alpha_{j}+\cdots+\alpha_{l-1}\right)+\alpha_{l} .
$$

\footnotetext{
${ }^{1}$ For $B_{3}$, a factor 2 appears in some brackets but this does not affect the final result.
} 
For $j \in\{3, \ldots, l\}$, set

$$
\beta_{j}^{\prime}:=\alpha_{2}+\cdots+\alpha_{j-1}, \quad \delta_{j}^{\prime}:=\alpha_{1}+\cdots+\alpha_{j-1}+2\left(\alpha_{j}+\cdots+\alpha_{l-1}\right)+\alpha_{l} .
$$

Then

$$
\forall j \in\{3, \ldots, l\}, \quad \beta_{j}+\delta_{j}=\beta_{j}^{\prime}+\delta_{j}^{\prime}=\theta_{0}=\frac{1}{2}\left(\theta+\theta_{1}\right) \quad \text { and } \quad \beta_{2}+\delta_{2}=\theta_{0} .
$$

We can choose a Chevalley basis of $\mathfrak{g}$ such that the vector

$$
\begin{aligned}
v_{2}:= & e_{\theta}(-1) e_{-\alpha_{1}}(-1)-\frac{1}{2} h_{1}(-1) e_{\theta_{0}}(-1)+e_{\theta_{0}}(-2) \\
& -e_{\beta_{2}}(-1) e_{\delta_{2}}(-1)-\frac{1}{2} \sum_{j=3}^{l}\left(e_{\beta_{j}}(-1) e_{\delta_{j}}(-1)-e_{\beta_{j}^{\prime}}(-1) e_{\delta_{j}^{\prime}}(-1)\right)
\end{aligned}
$$

is singular for $V^{k}(\mathfrak{g})$ with $k=-(l+6) / 2$. The verifications are left to the reader. This remark will be not used in the sequel.

Proof of Theorem 3.1. Let $\mathfrak{g}, k$ be as in Theorem. Then $\sigma\left(w_{i}\right)$ is a singular vector of $V^{k}(\mathfrak{g})$ for all $i$ by Theorem 4.2. Let $N$ be the submodule of $V^{k}(\mathfrak{g})$ generated by $\sigma\left(w_{i}\right)$ for all $i$, and set $\tilde{V}_{k}(\mathfrak{g})=V^{k}(\mathfrak{g}) / N$. By construction the image of $A(N)$ in $U(\mathfrak{g})$ is $\mathcal{J}_{W}$. Hence

$$
A\left(\tilde{V}_{k}(\mathfrak{g})\right)=U(\mathfrak{g}) / \mathcal{J}_{W} .
$$

It remains to show that $\tilde{V}_{k}(\mathfrak{g})=V_{k}(\mathfrak{g})$, that is, $\tilde{V}_{k}(\mathfrak{g})$ is simple. (In the case that $k$ is admissible, that is, if $\mathfrak{g}$ is of type $G_{2}, F_{4}$, this follows from [KW88]. Also, this has been proved in [Pe13] in the case that $\mathfrak{g}$ is of type $D_{4}$.)

Suppose that $\tilde{V}_{k}(\mathfrak{g})$ is not simple, or equivalently, $\tilde{V}_{k}(\mathfrak{g})$ is reducible as a $\widehat{\mathfrak{g}}-$ module. Then there is at least one non-zero weight singular vector, say, $v$. Let $\mu$ be the weight of $v$, and let $M$ be a submodule of $\tilde{V}_{k}(\mathfrak{g})$ generated by $v$. Since $M_{\text {top }}=L_{\mathfrak{g}}(\bar{\mu}), L_{\mathfrak{g}}(\bar{\mu})$ is a module over $A\left(\tilde{V}_{k}(\mathfrak{g})\right)=U(\mathfrak{g}) / \mathcal{J}_{W}=\mathbb{C} \oplus U(\mathfrak{g}) / \mathcal{J}_{0}$. On the other hand $L_{\mathfrak{g}}(\bar{\mu})$ is finite-dimensional since it is a submodule of $V^{k}(\mathfrak{g})_{d}$ for some $d$. This implies that $L_{\mathfrak{g}}(\bar{\mu})$ cannot be a $U(\mathfrak{g}) / \mathcal{J}_{0}$-module. Therefore $\bar{\mu}=0$. This implies that $v$ coincides with the highest weight vector of $\tilde{V}_{k}(\mathfrak{g})$ up to nonzero multiplication, which is a contradiction.

\section{Proof of Theorem 1.1}

Let $\mathfrak{g}$ be of type $D_{l}, l \geqslant 4, E_{6}, E_{7}$, or $E_{8}$.

For $n \in \mathbb{Z}_{\geqslant 0}$, set

$$
k_{n}= \begin{cases}n-h^{\vee} / 6-1 & \text { if } \mathfrak{g} \text { is of type } D_{4}, E_{6}, E_{7}, E_{8}, \\ n-2 & \text { if } \mathfrak{g} \text { is of type } D_{l}, l \geqslant 5 .\end{cases}
$$

Let $N$ be the submodule of $V^{k}(\mathfrak{g})$ generated by $\sigma\left(w_{i}\right)^{n+1}$ for all $i$ for type $D_{4}, E_{6}$, $E_{7}, E_{8}$, and by $\sigma\left(w_{1}\right)^{n+l-3}$ and $\sigma\left(w_{2}\right)^{n+1}$ for type $D_{l}, l \geqslant 5$, and let

$$
\tilde{V}_{k_{n}}(\mathfrak{g}):=V^{k_{n}}(\mathfrak{g}) / N \text {. }
$$

Conjecture 1. $\tilde{V}_{k_{n}}(\mathfrak{g})=V_{k_{n}}(\mathfrak{g})$, that is, $\tilde{V}_{k_{n}}(\mathfrak{g})$ is simple, if $k_{n}<0$. 
We have proven Conjecture 1 in the case that $n=0$ in type $D_{4}, E_{6}, E_{7}, E_{8}$ in the proof of Theorem 3.1.

Remark 5.1. If $k_{n} \geqslant 0, \tilde{V}_{k_{n}}(\mathfrak{g})$ is obviously not simple as the maximal submodule of $V^{k_{n}}(\mathfrak{g})$ is generated by $e_{\theta}(-1)^{k_{n}+1}$.

Proposition 5.2. For each $n \geqslant 0$, we have $X_{\tilde{V}_{k_{n}}(\mathfrak{g})}=\overline{\mathbb{O}_{\text {min }}}$.

Proof. Set $k=k_{n}$. The exact sequence $0 \rightarrow N \rightarrow V^{k}(\mathfrak{g}) \rightarrow \tilde{V}_{k}(\mathfrak{g}) \rightarrow 0$ induces an exact sequence

$$
N / \mathfrak{g}\left[t^{-1}\right] t^{-2} N \rightarrow V^{k}(\mathfrak{g}) / \mathfrak{g}\left[t^{-1}\right] t^{-2} V^{k}(\mathfrak{g}) \rightarrow \tilde{V}_{k}(\mathfrak{g}) / \mathfrak{g}\left[t^{-1}\right] t^{-2} \tilde{V}_{k}(\mathfrak{g}) \rightarrow 0 .
$$

Under the isomorphism $V^{k}(\mathfrak{g}) / \mathfrak{g}\left[t^{-1}\right] t^{-2} V^{k}(\mathfrak{g}) \cong S(\mathfrak{g})$, the image of $N / \mathfrak{g}\left[t^{-1}\right] t^{-2} N$ in $V^{k}(\mathfrak{g}) / \mathfrak{g}\left[t^{-1}\right] t^{-2} V^{k}(\mathfrak{g})$ is identified with the ideal $J$ of $S(\mathfrak{g})$ generated by some powers of $w_{i}$ for all $i$. Hence $J \subset J_{W} \subset \sqrt{J}$. Therefore,

$$
\sqrt{J}=\sqrt{J_{W}}=J_{0}
$$

by Lemma 2.1 as required.

Proof of Theorem 1.1. For $\mathfrak{g}$ of type $A_{1}, A_{2}, G_{2}, F_{4}$, the number $-h^{\vee} / 6-1$ is admissible, and the statement (1) of the theorem is a special case of [Ar15a, Theorem 5.14]. So let us assume that $\mathfrak{g}$ is of type $D_{l}, l \geqslant 4, E_{6}, E_{7}$, or $E_{8}$ as above. Since $V_{k_{n}}(\mathfrak{g})$ is a quotient of $\tilde{V}_{k_{n}}(\mathfrak{g})$, Proposition 5.2 implies that

$$
X_{V_{k_{n}}(\mathfrak{g})} \subset \overline{\mathbb{O}_{\min }}=\mathbb{O}_{\min } \cup\{0\} .
$$

Therefore $X_{V_{k_{n}}(\mathfrak{g})}$ is either $\{0\}$ or $\overline{\mathbb{O}_{\text {min }}}$. The assertion follows since $X_{V_{k}(\mathfrak{g})}=\{0\}$ if and only if $k \in \mathbb{Z}_{\geqslant 0}$ by [Ar15a, Proposition 4.25] (see also Theorem 6.1 (2) and (3) (a)).

The following assertion was proved in [Pe13] in the case that $\mathfrak{g}$ is of type $D_{4}$ and $k=-2$.

Corollary 5.3. Let $\mathfrak{g}, k$ be as in Theorem 1.1. Then $V_{k}(\mathfrak{g})$ has only finitely many simple modules in the category $\mathcal{O}$.

Proof. By [DSK06, Proposition 2.17(c)], [ALY14, Proposition 3.3] there is a surjection

$$
R_{V_{k}(\mathfrak{g})} \rightarrow \operatorname{gr} A\left(V_{k}(\mathfrak{g})\right)
$$

of Poisson algebras, where gr $A\left(V_{k}(\mathfrak{g})\right)$ is the associated graded algebra of $A\left(V^{k}(\mathfrak{g})\right)$ with respect to Zhu's filtration [Z96]. Hence Specm $\left(\operatorname{gr} A\left(V_{k}(\mathfrak{g})\right)\right) \subset X_{V_{k}(\mathfrak{g})} \subset \mathcal{N}$. It follows that the center $Z(\mathfrak{g})$ of $U(\mathfrak{g})$ acts finitely on $A\left(V_{k}(\mathfrak{g})\right)$ and therefore there are only finitely many possible central characters of simple modules of $A\left(V_{k}(\mathfrak{g})\right)$.

Remark 5.4. Let $\mathfrak{g}, f$ be as in Theorem 1.1. As in the same way as [Ar15b, Theorem 9.5], one finds that $X_{V_{k}(\mathfrak{g})}=\operatorname{Specm}\left(\operatorname{gr} A\left(V_{k}(\mathfrak{g})\right)\right)$, which gives another evidence for [Ar15b, Conjecture 1]. 
Conjecture 2. We have

$$
X_{V_{k}(\mathfrak{g})}=\overline{\mathbb{O}_{\min }}
$$

if and only if

(1) $\mathfrak{g}$ is of type $A_{1}$, and $k$ is a rational admissible number that is not an integer, or $k=-2$.

(2) $\mathfrak{g}$ is of type $A_{2}, C_{l}(l \geqslant 2), F_{4}$, and $k$ is admissible with denominator 2.

(3) $\mathfrak{g}$ is of type $G_{2}$, and $k$ is admissible with denominator 3 , or $k=-1$.

(4) $\mathfrak{g}$ is of type $D_{4}, E_{6}, E_{7}, E_{8}$ and $k$ is an integer such that

$$
-\frac{h^{\vee}}{6}-1 \leqslant k \leqslant-1 \text {. }
$$

(5) $\mathfrak{g}$ is of type $D_{l}$ with $l \geqslant 5$, and $k=-2,-1$.

One can easily verify Conjecture 2 for type $A_{1}$. Note that the "if" part of Conjecture 2 follows from Theorem 1.1 and [Ar15a, Theorem 5.14].

\section{Proof of Theorem 1.2}

Let $H_{f_{\theta}}^{\frac{\infty}{2}+\bullet}(M)$ denote the BRST cohomology associated with the quantized Drinfeld-Sokolov reduction corresponding to $f_{\theta}$ ([KRW03]), so that

$$
\mathcal{W}^{k}\left(\mathfrak{g}, f_{\theta}\right)=H_{f_{\theta}}^{\frac{\infty}{2}+0}\left(V^{k}(\mathfrak{g})\right)
$$

The correspondence $M \mapsto H_{f_{\theta}}^{\frac{\infty}{2}+0}(M)$ gives a functor $\mathcal{O}_{k} \rightarrow \mathcal{W}^{k}\left(\mathfrak{g}, f_{\theta}\right)$-Mod, where $\mathcal{O}_{k}$ is the category $\mathcal{O}$ of $\widehat{\mathfrak{g}}$ of level $k$ and $\mathcal{W}^{k}\left(\mathfrak{g}, f_{\theta}\right)$-Mod is the category of $\mathcal{W}^{k}\left(\mathfrak{g}, f_{\theta}\right)$ modules.

Recall that $\mathcal{W}_{k}\left(\mathfrak{g}, f_{\theta}\right)$ is the unique simple quotient of $\mathcal{W}^{k}\left(\mathfrak{g}, f_{\theta}\right)$.

Theorem 6.1. (1) ([Ar05, Main Theorem $])$ The functor $\mathcal{O}_{k} \rightarrow \mathcal{W}^{k}\left(\mathfrak{g}, f_{\theta}\right)$-Mod, $M \mapsto H_{f_{\theta}}^{\frac{\infty}{2}+0}(M)$, is exact.

(2) ([Ar05, Main Theorem]) We have $H_{f_{\theta}}^{\frac{\infty}{2}+0}(L(\lambda))=0$ if $\lambda\left(\alpha_{0}^{\vee}\right) \in \mathbb{Z}_{\geqslant 0}$, where $\alpha_{0}^{\vee}=K-\theta$. Otherwise $H_{f_{\theta}}^{\frac{\infty}{2}+0}(L(\lambda))$ is an irreducible highest weight representation of $\mathcal{W}^{k}\left(\mathfrak{g}, f_{\theta}\right)$. In particular,

$$
H_{f_{\theta}}^{\frac{\infty}{2}+0}\left(V_{k}(\mathfrak{g})\right) \cong \begin{cases}\mathcal{W}_{k}\left(\mathfrak{g}, f_{\theta}\right) & \text { if } k \notin \mathbb{Z}_{\geqslant 0}, \\ 0 & \text { if } k \in \mathbb{Z}_{\geqslant 0} .\end{cases}
$$

(3) ([Ar15a, Theorem 4.21]) For any quotient $V$ of $V^{k}(\mathfrak{g})$ we have

$$
X_{H_{f_{\theta}}^{\frac{\infty}{2}+0}(V)}=X_{V} \cap \mathcal{S}_{\min } .
$$

Hence

(a) ([Ar15a, Proposition 4.22]) $H_{f_{\theta}}^{\frac{\infty}{2}+0}(V) \neq 0$ if and only if $\overline{\mathbb{O}_{\min }} \subset X_{V}$.

(b) $\left(\left[\right.\right.$ Ar15a, Theorem 4.23]) $H_{f_{\theta}}^{\frac{\infty}{2}+0}(V)$ is a lisse vertex algebra if $X_{V}=$ $\overline{\mathbb{O}_{\min }}$. 
Remark 6.2. By [KW04, Theorem 6.3], the image $H_{f_{\theta}^{2}}^{\frac{\infty}{2}+0}(M(\lambda))$ of the Verma module $M(\lambda)$ of $\widehat{\mathfrak{g}}$ with highest weight $\lambda$ is isomorphic to a Verma module of $\mathcal{W}^{k}\left(\mathfrak{g}, f_{\theta}\right)$. Moreover, all the Verma modules of $\mathcal{W}^{k}\left(\mathfrak{g}, f_{\theta}\right)$ appear in this way. By Theorem $6.1(1),(2), H_{f_{\theta}}^{\frac{\infty}{2}+0}(L(\lambda))$ is the unique simple quotient of $H_{f_{\theta}}^{\frac{\infty}{2}+0}(M(\lambda))$ provided $\lambda\left(\alpha_{0}^{\vee}\right) \notin \mathbb{Z}_{\geqslant 0}$. From this, one sees that all the irreducible highest weight representations of $\mathcal{W}^{k}\left(\mathfrak{g}, f_{\theta}\right)$ appear as $H_{f_{\theta}}^{\frac{\infty}{2}+0}(L(\lambda))$ for some $\lambda$, see [Ar05] for the details.

Let $k$ be non-critical, that is, $k+h^{\vee} \neq 0$. By [KW04, Theorem 6.3], one finds that $H_{f_{\theta}}^{\frac{\infty}{2}+0}(M(\lambda)) \cong H_{f_{\theta}}^{\frac{\infty}{2}+0}(M(\mu))$ if and only if $\mu=s_{0} \circ \lambda$, where $s_{0}$ is the reflection corresponding to $\alpha_{0}$. It follows that $H_{f_{\theta}}^{\frac{\infty}{2}+0}(L(\lambda))$ and $H_{f_{\theta}}^{\frac{\infty}{2}+0}(L(\mu))$ are nonzero and isomorphic if and only if $\lambda\left(\alpha_{0}^{\vee}\right), \mu\left(\alpha_{0}^{\vee}\right) \notin \mathbb{Z}_{\geqslant 0}$ and $\mu=s_{0} \circ \lambda$.

Proof of Theorem 1.2. Let $k=k_{n}$ with $n \geqslant 0$ as in $\S 5$. We have shown that $X_{\tilde{V}_{k}(\mathfrak{g})}=\overline{\mathbb{O}_{\min }}$ in Proposition 5.2. Hence the vertex algebra $H_{f_{\theta}}^{\frac{\infty}{2}+0}\left(\tilde{V}_{k}(\mathfrak{g})\right)$ is nonzero and lisse by Theorem 6.1 (3). Note that both $\mathcal{W}_{k}\left(\mathfrak{g}, f_{\theta}\right)$ and $H_{f_{\theta}}^{\frac{\infty}{2}+0}\left(\tilde{V}_{k}(\mathfrak{g})\right)$ are quotients of $\mathcal{W}^{k}\left(\mathfrak{g}, f_{\theta}\right)$. Indeed, $H_{f_{\theta}}^{\frac{\infty}{2}+0}\left(\tilde{V}_{k}(\mathfrak{g})\right)$ is a quotient of $\mathcal{W}^{k}\left(\mathfrak{g}, f_{\theta}\right)=$ $H_{f_{\theta}}^{\frac{\infty}{2}+0}\left(V^{k}(\mathfrak{g})\right)$ by Theorem $6.1(1)$ since $\tilde{V}_{k}(\mathfrak{g})$ is a quotient of $V^{k}(\mathfrak{g})$. Because it is a unique simple quotient of $\mathcal{W}^{k}\left(\mathfrak{g}, f_{\theta}\right), \mathcal{W}_{k}\left(\mathfrak{g}, f_{\theta}\right)$ is a quotient of $H_{f_{\theta}}^{\frac{\infty}{2}+0}\left(\tilde{V}_{k}(\mathfrak{g})\right)$, which is lisse as we have just proved. Therefore $\mathcal{W}_{k}\left(\mathfrak{g}, f_{\theta}\right)$ is lisse as well.

Conjecture 3. Let $\mathfrak{g}$ and $k$ be as in Theorem 1.2. Then $H_{f_{\theta}}^{\frac{\infty}{2}+0}\left(\tilde{V}_{k}(\mathfrak{g})\right) \cong \mathcal{W}_{k}\left(\mathfrak{g}, f_{\theta}\right)$, where $\tilde{V}_{k}(\mathfrak{g})$ is defined above.

Remark 6.3. Let $\mathfrak{g}$ and $k$ be as in Theorem 1.2. Then $\mathcal{W}_{k}\left(\mathfrak{g}, f_{\theta}\right) \varsubsetneqq H_{f_{\theta}}^{\frac{\infty}{2}+0}(L(\lambda))$ for any irreducible admissible representation $L(\lambda)$ of $\widehat{\mathfrak{g}}$. Indeed, if $k \leqslant-1$ (resp. if $k \geqslant-1), L\left(k \Lambda_{0}\right)=V_{k}(\mathfrak{g})$ (resp. $\left.L\left(s_{0} \circ k \Lambda_{0}\right)\right)$ is the unique irreducible highest weight representation of $\widehat{\mathfrak{g}}$ such that $\mathcal{W}_{k}\left(\mathfrak{g}, f_{\theta}\right) \cong H_{f_{\theta}}^{\frac{\infty}{2}+0}(L(\lambda))$, see Remark 6.2. But $k \Lambda_{0}$ (resp. $s_{0} \circ k \Lambda_{0}$ ) is not an admissible weight since it is not regular dominant.

\section{Classification of lisse minimal $W$-algebras}

Theorem 7.1. (1) $\mathcal{W}_{k}\left(\mathfrak{s p}_{2 l}, f_{\theta}\right), l \geqslant 2$, is lisse if and only if $k$ is admissible with denominator 2 , that is, $k=p / 2$ and $p$ is an odd number equal to or greater than -1 .

(2) $\mathcal{W}_{k}\left(\mathfrak{s o}_{7}, f_{\theta}\right)$ is lisse if and only if $k$ is admissible with denominator 2 , that is, $k=p / 2$ and $p$ is an odd integer equal to or greater than -3 .

(3) $\mathcal{W}_{k}\left(\mathfrak{s o}_{2 l+1}, f_{\theta}\right), l \geqslant 4$, is never lisse.

(4) $\mathcal{W}_{k}\left(\mathfrak{s o}_{2 l}, f_{\theta}\right), l \geqslant 2$, is lisse if and only if $k$ is an integer equal to or greater than -2 .

(5) $\mathcal{W}_{k}\left(F_{4}, f_{\theta}\right)$ is lisse if and only if $k$ is admissible with denominator 2 , that is, $k=p / 2$ and $p$ is an odd number equal to or greater than -5 .

(6) $\mathcal{W}_{k}\left(E_{6}, f_{\theta}\right)$ is lisse if and only if $k$ is an integer equal to or greater than -3 .

(7) $\mathcal{W}_{k}\left(E_{7}, f_{\theta}\right)$ is lisse if and only if $k$ is an integer equal to or greater than -4 . 
(8) $\mathcal{W}_{k}\left(E_{8}, f_{\theta}\right)$ is lisse if and only if $k$ is an integer equal to or greater than -6 .

If $\mathcal{W}_{k}\left(\mathfrak{g}, f_{\theta}\right)=\mathbb{C}$, then it is obviously lisse. Hence it is natural to ask when $\mathcal{W}_{k}\left(\mathfrak{g}, f_{\theta}\right)=\mathbb{C}$. It turns out not every $W$-algebra admits one-dimensional representations.

Theorem 7.2. Suppose $\mathfrak{g}$ is not of type $A_{1}$. The following are equivalent:

(1) $\mathcal{W}^{k}\left(\mathfrak{g}, f_{\theta}\right)$ admits a (non-twisted or Ramond-twisted) one-dimensional representation,

(2) $\mathcal{W}_{k}\left(\mathfrak{g}, f_{\theta}\right)=\mathbb{C}$,

(3) (a) $\mathfrak{g}$ belongs to the Deligne exceptional series and $k=-h^{\vee} / 6-1$, or

(b) $\mathfrak{g}=\mathfrak{s p}_{2 l}, l \geqslant 2$, and $k=-1 / 2$.

Remark 7.3. If $\mathfrak{g}=\mathfrak{s l}_{2}$, then $f_{\theta}=f_{\text {reg }}$ is regular, $\mathcal{W}_{k}\left(\mathfrak{g}, f_{\theta}\right)=\mathcal{W}_{k}\left(\mathfrak{s l}_{2}, f_{\text {reg }}\right)$ is the simple Virasoro vertex algebra provided that $k \neq-2$, and the results are wellknown ${ }^{2}$. Namely,

- $\mathcal{W}_{k}\left(\mathfrak{s l}_{2}, f_{\text {reg }}\right)$ is lisse if and only if either $k+2=p / q$, with $p, q \in \mathbb{Z}_{\geqslant 0}$, $(p, q)=1$ and $p, q \geqslant 2$, or $k+2=0$ (cf. [Ar12]),

- $\mathcal{W}_{k}\left(\mathfrak{s l}_{2}, f_{\text {reg }}\right)=\mathbb{C}$ if and only if either $k+2=2 / 3$, or $k+2=3 / 2$, or $k+2=0$.

The rest of this section is devoted to the proof of Theorem 7.1 and Theorem 7.2. Let $\mathfrak{g}_{0}$ be the center of the reductive Lie algebra $\mathfrak{g}^{\natural}$, so that

$$
\mathfrak{g}^{\natural}=\bigoplus_{i \geqslant 0} \mathfrak{g}_{i} .
$$

Define an invariant bilinear form on $\mathfrak{g}_{i}, i \geqslant 0$, by

$$
(x \mid y)_{i}^{\natural}:=\left(k+\frac{h^{\vee}}{2}\right)(x \mid y)-\frac{1}{4}\left(\operatorname{tr}_{\mathfrak{g}(0)}(\operatorname{ad} x \operatorname{ad} y)\right),
$$

where $(\mid)$ is the normalized inner product of $\mathfrak{g}$ as before. Then there exists a polynomial $k_{i}^{\natural}$ of $k$ of degree 1 such that

$$
(\mid)_{i}^{\natural}=k_{i}^{\natural}(\mid)_{i},
$$

where $(\mid)_{i}$ is the normalized inner product of $\mathfrak{g}_{i}$, that is, $\left(\theta_{i} \mid \theta_{i}\right)=2$.

By [KW04, Theorem 5.1], we have an embedding

$$
\bigotimes_{i \geqslant 0} V^{k_{i}^{\natural}}\left(\mathfrak{g}_{i}\right) \hookrightarrow \mathcal{W}^{k}\left(\mathfrak{g}, f_{\theta}\right)
$$

of vertex algebras.

Lemma 7.4. (1) Suppose that $\mathcal{W}_{k}\left(\mathfrak{g}, f_{\theta}\right)$ is lisse. Then the value of $k_{i}^{\natural}$ for all $i \geqslant 1$ must be a nonnegative integer.

(2) Suppose that $\mathcal{W}^{k}\left(\mathfrak{g}, f_{\theta}\right)$ admits a (non-twisted or Ramond-twisted) onedimensional representation. Then the value of $k_{i}^{\natural}$ for all $i \geqslant 0$ must be zero.

\footnotetext{
${ }^{2}$ Note that $\mathcal{W}_{r-2}\left(\mathfrak{s l}_{2}, f_{\text {reg }}\right) \cong \mathcal{W}_{1 / r-2}\left(\mathfrak{s l}_{2}, f_{\text {reg }}\right)$ for any $r \in \mathbb{C}^{*}$
} 
Proof. (1) By [DM06], if a lisse vertex algebra $V$ contains a quotient of an affine vertex algebra as a vertex subalgebra, this quotient must be integrable. With $V=\mathcal{W}_{k}\left(\mathfrak{g}, f_{\theta}\right)$, we deduce that the simple quotient $V_{k_{i}^{\natural}}\left(\mathfrak{g}_{i}\right)$ must be integrable for any $i \geqslant 1$, that is, $k_{i}^{\natural}$ is a nonnegative integer for any $i \geqslant 1$.

(2) If $\mathcal{W}^{k}\left(\mathfrak{g}, f_{\theta}\right)$ admits a (non-twisted or Ramond-twisted) one-dimensional representation, by restriction we obtain that $V^{k_{i}^{\natural}}\left(\mathfrak{g}_{i}\right)$, for $i \geqslant 0$, admits a onedimensional representation. Hence $k_{i}^{\natural}=0$ for all $i \geqslant 0$.

Lemma 7.5. The reductive Lie algebras $\mathfrak{g}^{\natural}=\bigoplus_{i \geqslant 0} \mathfrak{g}_{i}$ and the polynomials $k_{i}^{\natural}$ are described in the below Tables 3 and 4.

\begin{tabular}{|c|c|c|c|c|c|c|}
\hline & $\mathfrak{s l}_{3}$ & $\mathfrak{s l}_{l+1}, l \geqslant 3$ & $\mathfrak{s p}_{2 l}, l \geqslant 2$ & $\mathfrak{s o}_{7}$ & $\mathfrak{s o}_{8}$ & $\mathfrak{s o}_{n}, n \geqslant 9$ \\
\hline $\mathfrak{g}^{\natural}$ & $\begin{array}{c}\mathfrak{g}_{0}, \\
\mathfrak{g}_{0} \cong \mathbb{C}\end{array}$ & $\begin{array}{c}\mathfrak{g}_{0} \oplus \mathfrak{g}_{1} \\
\mathfrak{g}_{0} \cong \mathbb{C}, \mathfrak{g}_{1} \cong \mathfrak{s l}_{l-1}\end{array}$ & $\begin{array}{c}\mathfrak{g}_{1} \\
\mathfrak{g}_{1} \cong \mathfrak{s p}_{2 l-2}\end{array}$ & $\begin{array}{c}\mathfrak{g}_{1} \oplus \mathfrak{g}_{2} \\
\mathfrak{g}_{1} \cong \mathfrak{g}_{2} \cong \mathfrak{s l}_{2}\end{array}$ & $\begin{array}{l}\bigoplus_{i=1}^{3} \mathfrak{g}_{i} \\
\mathfrak{g}_{i} \cong \mathfrak{s l}_{2}\end{array}$ & $\begin{array}{c}\mathfrak{g}_{1} \oplus \mathfrak{g}_{2} \\
\mathfrak{g}_{1} \cong \mathfrak{s l}_{2}, \mathfrak{g}_{2} \cong \mathfrak{s o}_{n-4}\end{array}$ \\
\hline$k_{i}^{\natural}$ & $k_{0}^{\natural}=k+\frac{3}{2}$ & $\begin{array}{c}k_{0}^{\natural}=k+\frac{l+1}{2}, \\
k_{1}^{\natural}=k+1\end{array}$ & $k_{1}^{\natural}=k+\frac{1}{2}$ & $\begin{array}{l}k_{1}^{\natural}=k+\frac{3}{2}, \\
k_{2}^{\natural}=2 k+4\end{array}$ & $\begin{array}{l}k_{i}^{\natural}=k+2, \\
i \in\{1,2,3\}\end{array}$ & $\begin{array}{c}k_{1}^{\natural}=k+\frac{n}{2}-2, \\
k_{2}^{\natural}=k+2\end{array}$ \\
\hline
\end{tabular}

TABLE 3. $\mathfrak{g}^{\natural}=\bigoplus_{i \geqslant 0} \mathfrak{g}_{i}$ and $k_{i}^{\natural}$ for the classical types

\begin{tabular}{|c|c|c|c|c|c|}
\hline & $G_{2}$ & $F_{4}$ & $E_{6}$ & $E_{7}$ & $E_{8}$ \\
\hline $\mathfrak{g}^{\natural}$ & $\mathfrak{s l}_{2}$ & $\mathfrak{s p}_{6}$ & $\mathfrak{s l}_{6}$ & $\mathfrak{s o}_{12}$ & $E_{7}$ \\
$k_{1}^{\natural}$ & $3 k+5$ & $k+\frac{5}{2}$ & $k+3$ & $k+4$ & $k+6$ \\
\hline
\end{tabular}

TABLE 4. $\mathfrak{g}^{\natural}=\bigoplus_{i \geqslant 0} \mathfrak{g}_{i}$ and $k_{i}^{\natural}$ for the exceptional types

Proof. The verifications are easy and left to the reader.

Proof of Theorem \%.1. The "if" part of Theorem 7.1 has been already proven in Theorem 1.2 and [Ar15a, Theorem 5.18], and the "only if" part follows from Lemmas 7.4 and 7.5 .

Remark 7.6. For $\mathfrak{g}=\mathfrak{s p}_{2 l}$ it is possible to show the following.

$$
A\left(V_{-1 / 2}(\mathfrak{g})\right) \cong U(\mathfrak{g}) / \mathcal{J}_{W_{1}} \cong \mathbb{C} \times\left(L_{\mathfrak{g}}\left(\varpi_{1}\right)^{*} \otimes_{\mathbb{C}} L_{\mathfrak{g}}\left(\varpi_{1}\right)\right) \times U(\mathfrak{g}) / \mathcal{J}_{0},
$$

where $\mathcal{J}_{W_{1}}$ is the ideal generated by $W_{1}:=L_{\mathfrak{g}}\left(\theta+\theta_{1}\right) \subset W$. This implies that $\mathcal{J}_{0}$ is generated by $\mathcal{J}_{W_{1}}$ and $\Omega-c_{0}$.

Conjecture 4. (1) $\mathcal{W}_{k}\left(\mathfrak{s l}_{3}, f_{\theta}\right)$ is lisse if and only if $k$ is admissible with denominator 2 , that is, $k=p / 2$ and $p$ is an odd integer equal or greater than -3 .

(2) $\mathcal{W}_{k}\left(\mathfrak{s l}_{n}, f_{\theta}\right), n \geqslant 4$, is never lisse.

(3) $\mathcal{W}_{k}\left(G_{2}, f_{\theta}\right)$ is lisse if and only if $k$ is admissible with denominator 3 , or an integer equal to or greater than -1 . 
The "if" part of Conjecture 4 follows from [Ar15a, Theorem 5.18].

Proof of Theoren 7.2. Clearly (2) implies (1). The direction (1) $\Rightarrow(3)$ follows from Lemmas 7.4 and 7.5 .

Let us show (3) implies (2).

The $A_{2}$ case follows from [Ar13].

Assume that $\mathfrak{g}$ is of type $D_{l}, E_{6}, E_{7}$, or $E_{8}$. Note that $k=k_{0}$ in (3). Let $N$ be the submodule of $V^{k}(\mathfrak{g})$ generated by $v_{i}=\sigma\left(w_{i}\right)$, for all $i$, and set $\tilde{V}_{k}(\mathfrak{g})=V^{k}(\mathfrak{g}) / N$ as in Section 5. By Theorem 6.1 (1) we have an exact sequence

$$
0 \rightarrow H_{f_{\theta}}^{\frac{\infty}{2}+0}(N) \rightarrow H_{f_{\theta}}^{\frac{\infty}{2}+0}\left(V^{k}(\mathfrak{g})\right) \rightarrow H_{f_{\theta}}^{\frac{\infty}{2}+0}\left(\tilde{V}_{k}(\mathfrak{g})\right) \rightarrow 0
$$

of $\mathcal{W}^{k}\left(\mathfrak{g}, f_{\theta}\right)$-modules. The image $\bar{v}_{i}$ of $v_{i} \in N$ in $H_{f_{\theta}}^{\frac{\infty}{2}+0}\left(V^{k}(\mathfrak{g})\right)=\mathcal{W}^{k}\left(\mathfrak{g}, f_{\theta}\right)$ is nonzero, since its image in $R_{\mathcal{W}_{k}\left(\mathfrak{g}, f_{\theta}\right)}=\mathbb{C}\left[\mathcal{S}_{\text {min }}\right]$ is nonzero and coincides with $e_{\theta_{i}}$ under the identification $\mathbb{C}\left[\mathcal{S}_{\text {min }}\right]=S\left(\mathfrak{g}^{f_{\theta}}\right)$, where $e_{\theta_{i}}$ is the highest root vector of $\mathfrak{g}_{i}$. By weight consideration one finds that $\bar{v}_{i}$ coincides with $e_{\theta_{i}}(-1) \in V^{k_{i}^{\natural}}\left(\mathfrak{g}_{i}\right) \subset$ $\mathcal{W}^{k}\left(\mathfrak{g}, f_{\theta}\right)$ up to non-zero constant multiplication.

Since $\mathcal{W}^{k}\left(\mathfrak{g}, f_{\theta}\right)_{1}=\mathfrak{g}^{\natural}=\bigoplus_{i \geqslant 1} \mathfrak{g}_{i}$, the whole weight one space $\mathcal{W}^{k}\left(\mathfrak{g}, f_{\theta}\right)_{1}$ is included in the image of $H_{f_{\theta}}^{\frac{\infty}{2}+0}(N)$. Then from the commutation relations of $\mathcal{W}^{k}\left(\mathfrak{g}, f_{\theta}\right)$ described in [KW04, Theorem 5.1] it follows that all the generators $G^{v}$, $v \in \mathfrak{g}_{1 / 2}$, defined in [KW04], and the conformal vector are also in the image of $H_{f_{\theta}}^{\frac{\infty}{2}+0}(N)$. Therefore $H_{f_{\theta}}^{\frac{\infty}{2}+0}\left(\tilde{V}_{k}(\mathfrak{g})\right)$ must be trivial, and hence, so is its simple quotient $\mathcal{W}_{k}\left(\mathfrak{g}, f_{\theta}\right)$.

Assume that $\mathfrak{g}$ is of type $C_{l}, G_{2}$ or $F_{4}$, so that $\mathfrak{g}^{\natural}$ is simple and $k$ is admissible, and hence the maximal submodule $N_{k}$ of $V^{k}(\mathfrak{g})$ is generated by a singular vector $v$. By Theorem 6.1 (1), (2) we have the exact sequence

$$
0 \rightarrow H_{f_{\theta}}^{\frac{\infty}{2}+0}(N) \rightarrow \mathcal{W}^{k}\left(\mathfrak{g}, f_{\theta}\right) \rightarrow \mathcal{W}_{k}\left(\mathfrak{g}, f_{\theta}\right) \rightarrow 0 .
$$

Also, by [KW04, Theorem 6.3.1] $H_{f_{\theta}}^{\frac{\infty}{2}+0}(N)$ is generated by the image $\bar{v}$ of $v$. Since the image of $H_{f_{\theta}}^{\frac{\infty}{2}+0}(N)$ in $\mathcal{W}^{k}\left(\mathfrak{g}, f_{\theta}\right)$ is nonzero as $\mathcal{W}_{k}\left(\mathfrak{g}, f_{\theta}\right)$ is lisse [Ar15a], the image of $\bar{v}$ in $\mathcal{W}^{k}\left(\mathfrak{g}, f_{\theta}\right)$ is nonzero. Hence, as in the same manner as above, by weight consideration it follows that $\mathcal{W}^{1}\left(\mathfrak{g}, f_{\theta}\right)_{1}$ is included in the image of $H_{f_{\theta}}^{\frac{\infty}{2}+0}(N)$, which gives that $\mathcal{W}_{k}\left(\mathfrak{g}, f_{\theta}\right)=\mathbb{C}$ as required.

\section{REFERENCES}

[Ad94] Dražen Adamović. Some rational vertex algebras. Glas. Mat. Ser. III, 29(49)(1):25-40, 1994.

[Ad97] Dražen Adamović. Vertex operator algebras and irreducibility of certain modules for affine Lie algebras. Math. Res. Lett. 4(6):809-821, 1997.

[AM95] Dražen Adamović and Antun Milas. Vertex operator algebras associated to modular invariant representations for $A_{1}^{(1)}$. Math. Res. Lett., 2(5):563-575, 1995.

[Ar05] Tomoyuki Arakawa. Representation theory of superconformal algebras and the KacRoan-Wakimoto conjecture. Duke Math. J., 130(3):435-478, 2005.

[Ar12] Tomoyuki Arakawa. A remark on the $C_{2}$ cofiniteness condition on vertex algebras. Math. Z., 270(1-2):559-575, 2012.

[Ar13] Tomoyuki Arakawa. Rationality of Bershadsky-Polyakov vertex algebras. Comm. Math. Phys., 323(2):627-633, 2013. 
[Ar15a] Tomoyuki Arakawa. Associated varieties of modules over Kac-Moody algebras and $C_{2}$ cofiniteness of $W$-algebras. Int. Math. Res. Not.(2015) Vol. 2015 11605-11666.

[Ar15b] Tomoyuki Arakawa. Rationality of $W$-algebras: principal nilpotent cases. Ann. Math., 182(2):565-694, 2015.

[Ar16] Tomoyuki Arakawa. Rationality of admissible affine vertex algebras in the category $\mathcal{O}$. Duke Math. J., 165(1), 67-93, 2016.

[ALY14] Tomoyuki Arakawa, Ching Hung Lam, and Hiromichi Yamada. Zhu's algebra, $C_{2}{ }^{-}$ algebra and $C_{2}$-cofiniteness of parafermion vertex operator algebras. Adv. Math., 264:261-295, 2014.

[AL11] Jonathan D. Axtell and Kyu-Hwan Lee. Vertex operator algebras associated to type $G$ affine Lie algebras. J. Algebra, 337:195-223, 2011.

$\left[\mathrm{BLL}^{+} 15\right]$ Christopher Beem, Madalena Lemos, Pedro Liendo, Wolfger Peelaers, Leonardo Rastelli, and Balt C. van Rees. Infinite chiral symmetry in four dimensions. Comm. Math. Phys., 336(3):1359-1433, 2015.

[BK01] Bojko Bakalov and Alexander Kirillov, Jr. Lectures on tensor categories and modular functors, volume 21 of University Lecture Series. American Mathematical Society, Providence, RI, 2001.

[BD] Alexander Beilinson and Vladimir Drinfeld. Quantization of Hitchin's integrable system and Hecke eigensheaves. preprint.

[CR12] Thomas Creutzig and David Ridout. Modular data and Verlinde formulae for fractional level WZW models I. Nuclear Phys. B, 865(1):83-114, 2012.

[CR13] Thomas Creutzig and David Ridout. Modular data and Verlinde formulae for fractional level WZW models II. Nuclear Phys. B, 875(2):423-458, 2013.

[D96] Pierre Deligne. La série exceptionnelle de groupes de Lie. C. R. Acad. Sci. Paris, Ser I, 322(4), 321-326, 1996.

[DG02] Pierre Deligne, and Benedict Gross. On the exceptional series, and its descendants. C. R. Acad. Sci. Paris, Ser I, 335(11), 877-881, 2002.

[DSK06] Alberto De Sole and Victor G. Kac. Finite vs affine $W$-algebras. Japan. J. Math., 1(1):137-261, 2006.

[DM06] Chongying Dong and Geoffrey Mason. Integrability of $C_{2}$-cofinite vertex operator algebras. Int. Math. Res. Not., pages Art. ID 80468, 15, 2006.

[FM97] Boris Feigin and Fyodor Malikov. Modular functor and representation theory of $\widehat{\mathrm{sl}}_{2}$ at a rational level. In Operads: Proceedings of Renaissance Conferences (Hartford, CT/Luminy, 1995), volume 202 of Contemp. Math., pages 357-405, Providence, RI, 1997. Amer. Math. Soc.

[FBZ04] Edward Frenkel and David Ben-Zvi. Vertex algebras and algebraic curves, volume 88 of Mathematical Surveys and Monographs. American Mathematical Society, Providence, RI, second edition, 2004.

[FZ92] Igor Frenkel and Yongchang Zhu. Vertex operator algebras associated to representations of affine and Virasoro algebras. Duke Math. J., 66(1):123-168, 1992.

[GS04] Wee Teck Gan and Gordan Savin. Uniqueness of Joseph ideal. Math. Res. Lett., 11(56):589-597, 2004.

[G82] Devra Garfinkle. A new construction of the Joseph ideal. PhD thesis, MIT, 1982.

[H08] Yi-Zhi Huang. Rigidity and modularity of vertex tensor categories. Commun. Contemp. Math., 10(suppl. 1):871-911, 2008.

[J76] Anthony Joseph. The minimal orbit in a simple Lie algebra and its associated maximal ideal. Ann. Sci. École Norm. Sup. (4), 9(1):1-29, 1976.

[J98] Anthony Joseph. Orbital varietes of the minimal orbit. Ann. Sci. École Norm. Sup. (4), 31(1):17-45, 1998.

[KRW03] Victor Kac, Shi-Shyr Roan, and Minoru Wakimoto. Quantum reduction for affine superalgebras. Comm. Math. Phys., 241(2-3):307-342, 2003. 
[KW88] Victor Kac and Minoru Wakimoto. Modular invariant representations of infinitedimensional Lie algebras and superalgebras. Proc. Nat. Acad. Sci. U.S.A., 85(14):49564960, 1988.

[KW89] Victor Kac and Minoru Wakimoto. Classification of modular invariant representations of affine algebras. In Infinite-dimensional Lie algebras and groups (Luminy-Marseille, 1988), volume 7 of Adv. Ser. Math. Phys., pages 138-177. World Sci. Publ., Teaneck, NJ, 1989.

[KW04] Victor Kac and Minoru Wakimoto. Quantum reduction and representation theory of superconformal algebras. Adv. Math., 185(2):400-458, 2004.

[KW08] Victor Kac and Minoru Wakimoto. On rationality of $W$-algebras. Transform. Groups, 13(3-4):671-713, 2008.

[K15] Kazuya Kawasetsu. $\mathcal{W}$-algebras with non-admissible levels and the Deligne exceptional series. arXiv:1505.06985.

[M04] Masahiko Miyamoto. Modular invariance of vertex operator algebras satisfying $\mathrm{C}_{2}$ cofiniteness. Duke Math. J., 122(1):51-91, 2004.

[Pe07a] Ozren Perše. Vertex operator algebras associated to type $B$ affine Lie algebras on admissible half-integer levels. J. Algebra, 307(1):215-248, 2007.

[Pe07b] Ozren Perše. Vertex operator algebra analogue of embedding of $B_{4}$ into $F_{4}$. J. Pure Appl. Algebra, 211(3):702-720, 2007.

[Pe13] Ozren Perše. A note on representations of some affine vertex algebras of type D. Glas. Mat. Ser. III, 48(68)(1):81-90, 2013.

[Pr02] Alexander Premet. Special transverse slices and their enveloping algebras. Adv. Math., 170(1):1-55, 2002. With an appendix by Serge Skryabin.

[Pr07] Alexander Premet. Enveloping algebras of Slodowy slices and the Joseph ideal. J. Eur. Math. Soc., 9(3):487-543, 2007.

[W99] Weiqiang Wang. Dimension of a minimal nilpotent orbit. Proc. Amer. Math. Soc. 127(3):935-936, 1999.

[Z96] Yongchang Zhu. Modular invariance of characters of vertex operator algebras. J. Amer. Math. Soc., 9(1):237-302, 1996.

Research Institute for Mathematical Sciences, Kyoto University, Kyoto 606-8502 JAPAN

E-mail address: arakawa@kurims.kyoto-u.ac.jp

Laboratoire de Mathématiques et Applications, Téléport 2 - BP 30179, Boulevard

Marie et Pierre Curie, 86962 Futuroscope Chasseneuil Cedex, France

E-mail address: anne.moreau@math.univ-poitiers.fr 